\title{
Deformation of the Catheter and 3D Blood Vessel Model for a VR-based Catheter System
}

\author{
${ }^{* 1}$ Department of Intelligent Mechanical Systems Engineering \\ ${ }^{*}$ Graduate School of Engineering \\ Kagawa University \\ Hayashi-cho, Takamatsu, 761-0396, Japan \\ \{s12g591,s12d503\}@stmail.eng.kagawa-u.ac.jp
}

Shuxiang $\mathrm{Guo}^{{ }^{*}{ }^{* 3}} \mathrm{Mohan}_{\mathrm{Qu}}{ }^{{ }_{2}}$ Baofeng $\mathrm{Gao}^{* 3}$ and $\mathrm{Jin}_{\mathrm{Guo}}{ }^{{ }_{2}}$

\begin{abstract}
There is an increasing realization that Minimally Invasive Surgery (MIS) is a specialized surgical technique that permits vascular interventions through very small incisions. Some operational tasks can be performed significantly better by robots than humans due to associated hazards and distance. However, the significant disadvantage of this surgical technique is its complexity; therefore, extensive training before surgery is necessary. At present, the operator can only determine the required action by visually examining in remote side which limits the task. In this paper, we present virtual reality technology based simulators for doctor training with force feedback in minimally invasive surgery. The simulators allow generating realistic physical-based model of the catheter and blood vessels, and enabling surgeons to touch, feel and manipulate the virtual catheter inside vascular model according to the same surgical operation mode used in actual MIS. The design of the VR system and initial experimental results are presented in experiments part of this paper. And the experimental results show that the error rate varies among an acceptable range and the simulators can be used for surgical training.
\end{abstract}

Index Terms -Virtual Reality based Robotic Catheter System, Catheter, Minimally Invasive Surgery (MIS).

\section{INTRODUCTION}

Significant research effects have been done in the development of technology for minimally invasive surgery (MIS), such as laparoscopy, has grown as a very suitable domain for robotic system [1][2]. Vascular intervention is expected to become increasingly popular in the medical practice, both for diagnosis and for surgery. A lot of diagnosis and medical surgery with an endoscope or a catheter are performed for minimum invasive surgery recently. [3][4] However, it requires a lot of skills for the operation in order to do the operation inside the human body that cannot be watched directly.

Because minimally invasive techniques have unavoidable reduced the sense of touch compared to open surgery, surgeons have to rely more on the haptic feeling generated by the interaction between blood vessels and the catheter.[5][11] Even if the color and texture of blood vessels convey crucial anatomical information visually, touch is still critical in the surgeries. The benefits of using haptic feedback

\author{
${ }^{* 3}$ Beijing Institute of Technology \\ 5 South Zhongguancun Street, Haidian District, Beijing, \\ 100081, China \\ guo@eng.kagawa-u.ac.jp \\ gbfbob_81@163.com
}

devices in minimally invasive surgery training through simulation have already been recognized by several research groups and many of companies working in this area [12][20]. However, in these researches, the virtual surgical training was carried out without haptic feedback or researched on the virtual model of body organ without the physical based vascular model. Moreover, some achievements in this area used Phantom Omni or other haptic devices as a controller to operate the virtual minimally invasive surgery [20]-[26]. In addition, some commercial robotic surgical systems have been developed in recent years. One of products that have been developed is a Sensei Robotic Catheter System offered by Hansen Medical which can provide the physician more stability and more force information in catheter placement with the Artisan sheath compared to manual techniques. It also allows for more precise manipulation with less radiation exposure to the doctor, commensurate with higher procedural complications to the patient. Because of the sheath's multiple degrees of freedom, force detection at the distal tip is very hard. Catheter Robotics Inc. has developed a remote catheter system called Amigo. This system has a robotic sheath to steer catheters which is controlled at a nearby work station, in a manner similar to the Sensei system. The first in human use of this system was in April 2010 in Leicester UK, where it was used to ablate artificial flutter. Magnatecs Inc. has produced their 'Catheter Guidance Control and Imaging' (CGCI) system. This has 4 large magnets placed around the table, with customized catheters containing magnets in the tip. The catheter is again moved by the magnetic fields and is controlled at a nearby work station. The system facilitates precise vector based navigation of magnetically-enabled guide wires for percutaneous coronary intervention (PCI) by using two permanent magnets located on opposite sides of the table to produce a controllable magnetic field. Nevertheless, it is not convenient when surgeon drive the catheter for inserting and rotating because it does not accord with the custom of surgeons' operations. 
The objective of this paper is to present the virtual reality simulators based on a novel robotic catheter operating system for surgeons' training in minimally invasive surgery. The simulators can not only generate the realistic virtual reality environment of blood vessels according to patient's special computed tomography (CT) or magnetic resonance imaging (MRI) files but also provide physical responses to the unskilled doctors. In addition, the controller allows simulating surgeon's operating skills to insert and rotate the catheter like surgeon operates catheter directly and carry out the intervention with haptic interfaces with force feedback, which provides the surgeon with a sense of touch.

This paper is organized as follows. Section II introduces the concept of our virtual reality based robotic catheter training system. And section III presents the algorithms and implementations of cerebrovascular model, catheter model and the interaction between cerebrovascular vessels and catheter. Then section IV discusses the results of our virtual reality simulators. Finally, section $\mathrm{V}$ describes our further directions, and comes to the conclusion of this paper.

\section{THE VIRTUAL REALITy BASED RoBotic CATHETER ASISTED SYSTEM}

The concept of our virtual reality based training system for minimally invasive surgery has been shown in Fig. 1 and Fig. 2 and Fig.3. The surgeons manipulate the right handle to move forward and backward, or to rotate the right handle during operation in master side. In the master side, the surgeon operates the handle to drive the catheter for inserting and rotating to clamp catheter directly, the control commands of the catheter operating system were transmitted to the virtual reality environment with a small time delay. After the VR system receiving the control commands from master side, the mechanism in a virtual reality environment clamps the catheter to insert and rotate inside the blood vessel model. If the catheter contact with the blood vessel wall, the contacting information can be calculated according to the deformation of vascular model. Then the surgeon can decide whether to insert or rotate the catheter depending on the information of

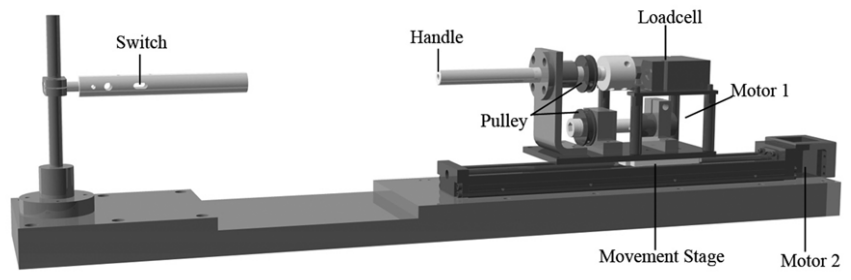

force feedback and the visual information.

Fig.1 The structure of the surgeon console

Fig.1 shows the surgeon console of the RCMS. The surgeon console is the master side of the whole system and it is operated by the experienced surgeons. Surgeons carry out operations by using the console. A switch placed on the left handle is used to control these two graspers in the catheter manipulator side; only one switch is enough because the catheter is clamped by one grasper at the same time. Surgeon's action is detected by using the right handle. The movement part of catheter manipulator keeps the same motion with the right handle of the console. The right handle can measure two actions of the surgeon's hand, one is axial movement and the other one is rotational movement. The handle is sustained by a bearing, and is linked to a loadcell; a pulley is fixed on the handle. A DC motor (Motor1) with encoder is applied to generate torque feedback. A pulley which is a couple of the upper one is fixed to the axle of the motor. All these parts are placed on a moving stage driven by a stepping motor (Motor2). Our novel robotic catheter operating system perfectly solves the operating precision of a surgeon, which is not well solved in the previous device, and the precision of inserting operation is $0.003 \mathrm{~mm}$. Another advantage of the developed system is that the operations in the master side accord with the custom of surgeon's operations in actual surgery. In addition, it can provide the force feedback to surgeons who operate the handle on the master side. Consequently, we developed the virtual reality simulators for surgical training based on the master side of robotic catheter operating system. For this system, we transmit the DICOM3.0 image to the master side, the camera image show us the external information and the DICOM image show us the inner information of the sick. The Surgeon in the master remote side can carry out the operation according to the two kinds of 3D image and complete the next operation step. However, the DICOM 3D image should be converted by us, and it is our important work to overcome so many unpredictable factors and difficulties.

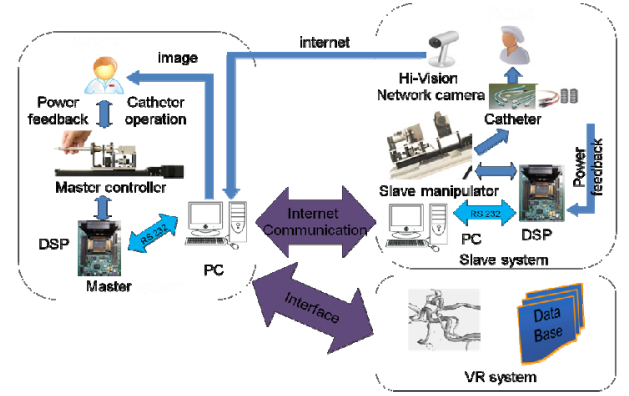

Fig.2 The Structure of the Virtual Reality based Robotic Catheter System

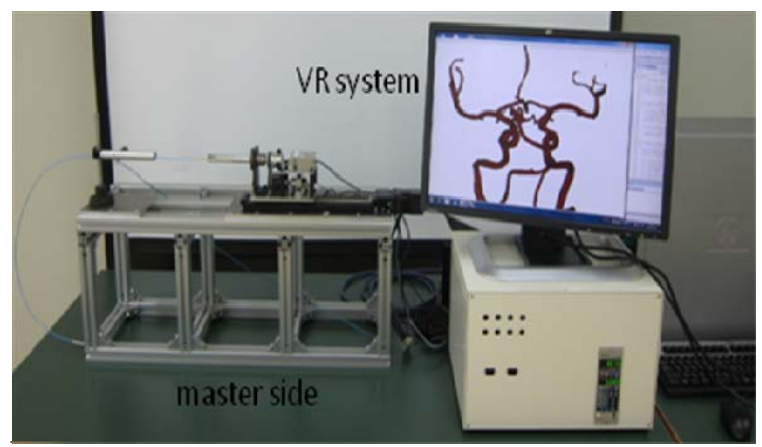

Fig.3 The virtual reality training system 


\section{PHYSICS-BASED MODELING OF 3D VESSEL}

A core component of a virtual reality surgical simulators and training system is realistic physical-based vascular models which are the virtual representations of real blood vessels that display accurate displacement and force response. To develop these models, the shape and the material properties of blood vessels should be measured and characterized in living conditions and in their native locations. Models with incorrect material properties and shape could result in adverse training effects. In order to reconstruct the realistic three dimension vascular model, we apply the median filter algorithm to reduce the noise of the CT images, then use local shareholding algorithm to realize the image segmentation of CT images, finally adopt the volume rendering technology to reconstruct the vascular model.

First, we used the software Cmake to install the DCMTK to the $\mathrm{VC}++$ package and then installed "DCMTK 3.5.4source code packages", "DCMTK 3.5.4-support libraries for windows", "Cmake 2.8.4 (one of Packages compiled tools)". Then we can use DCMTK to complete the image segmentation. Image grey value calculation formula can be shown in equation (1). According to the CT value of the DCM file which can present different parts of the human body, it is possible for us to change the grey value in the program and realize image segmentation.

$$
\mathrm{G}(V)=\left\{\begin{array}{c}
0, \quad \mathrm{~V}<C-\frac{W}{2} \\
\frac{g_{m}}{W}\left(V+\frac{W}{2}-C\right), \quad C-\frac{W}{2} \leq V \leq C+\frac{W}{2} \\
g_{m} \quad V>C+\frac{W}{2}
\end{array}\right.
$$

Where V means image data, $G(v)$ means Value displayed, $g_{m}$ is the maximum value displayed, $\mathrm{w}$ is the display window wide, and $\mathrm{c}$ is the window level.

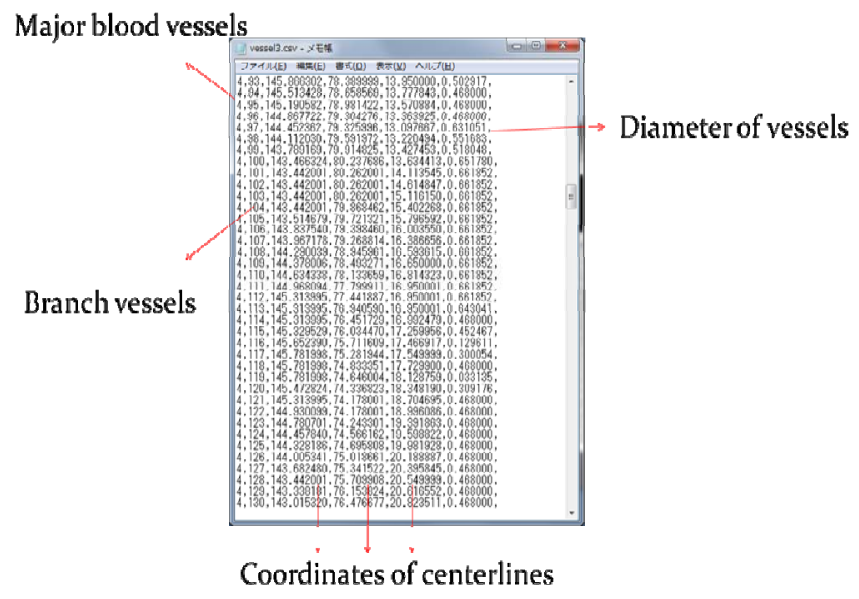

Fig.4 The Century line of the vessel model

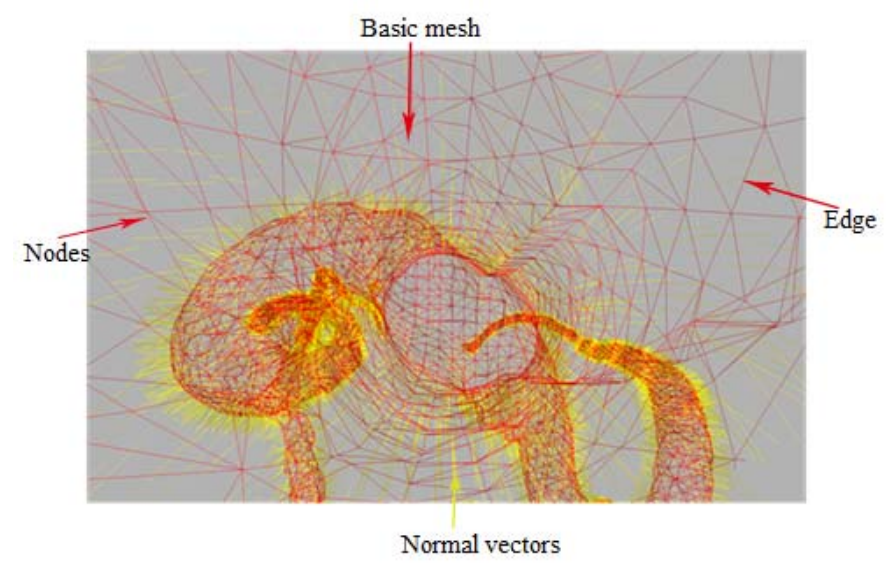

Fig.5 The mesh method of the 3D vessel model

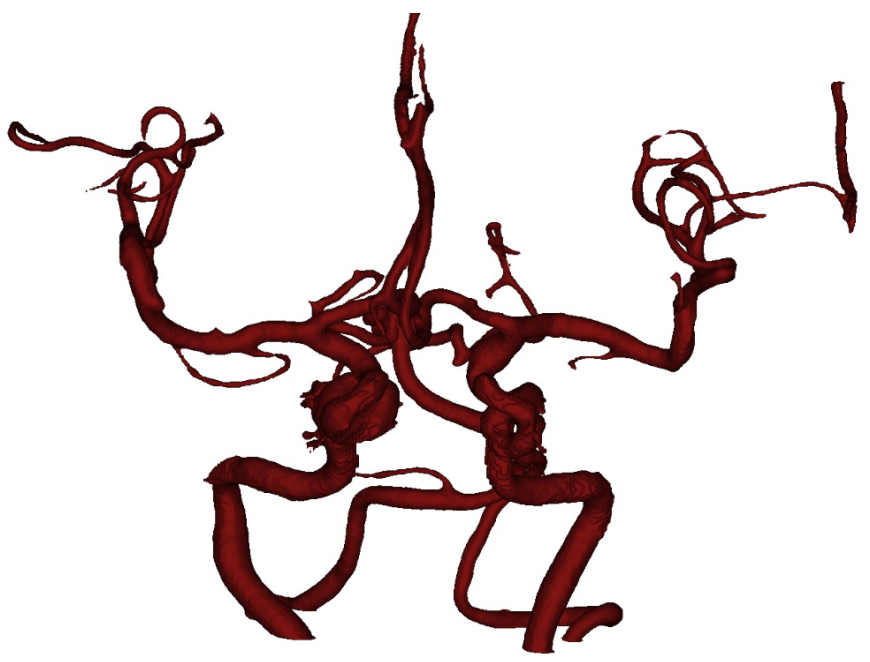

(a) Multi-branched blood vessels

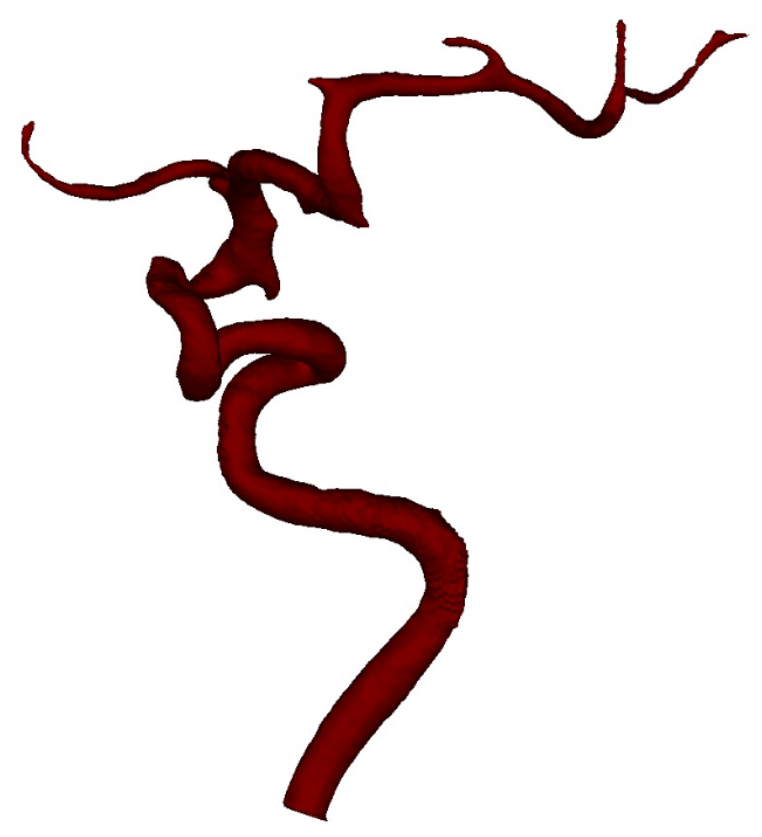

(b) A part of them as research topic

Fig.6 The mesh method of the 3D vessel model 
Fig4 shows the century line of the vessel model and Fig.5 show the mesh method of the 3D vessel model. Volume rendering methods generate images of a $3 \mathrm{D}$ volumetric data set without explicitly extracting geometric surfaces from the data. These techniques use an optical model to map data values to optical properties, such as colour and opacity. During rendering, optical properties are accumulated along each viewing ray to form an image of the data. We use texture mapping to apply images, or textures, to geometric objects. Volume aligned texturing produces images of reasonable quality, though there is often a noticeable transition when the volume is rotated. The three-dimension reconstruction images of the blood vessels have been shown in Fig. 6: (a) for the multi-branched blood vessels and we can choose a part of them as research topic shown in (b).

In order to realize the physical characteristics of the blood vessel model, we used mass-spring model to simulate the behaviors of the vascular structure. The mass-spring model is a widely used mesh-free method in surgical simulation, which models the object as masses connected to each other with springs and dampers. Every mass is represented respectively by its own coordinate, acceleration and velocity and deforms under the influence of inertial, the spring and damping forces and the forces applied by the surgical catheter. Hooke's law describes this force by a "spring equation" (1). F is the resulting force, $\mathrm{k}$ is the stiffness of the spring, $\mathrm{l}_{\mathrm{ij}}$ is the length of the spring connecting $\mathrm{i}$-th and $\mathrm{j}$-th particle while the zero superscript again denotes the rest pose.

$$
F_{i j}=k\left(\left|l_{i j}\right|-\left|l_{i j}{ }^{0}\right|\right) \frac{l_{i j}}{\left|l_{i j}\right|}
$$

The movement of the particles can be described by Newtonian mechanics. When only one spring and one particle is accounted for, it takes the form of equation (2), where $m$ is the mass of the observed particle, $\mathrm{c}$ is the damping coefficient of the spring, $\mathrm{k}$ is again the stiffness coefficient and $\mathrm{x}$ is the position of the particle, with appropriate time derivatives.

$$
m \ddot{x}+c \dot{x}+k x=0
$$

Equation(3) shows the actual form that needs to be solved for every particle $\mathrm{i}$ in a general MSS, with $\mathrm{F}^{\mathrm{e}}$ representing external forces acting on the particle, $F_{i j}$ the force computed using equation (1) and $\mathrm{N}_{\mathrm{i}}$ the set of particles, to which particle $i$ is connected by a spring.

$$
m_{i} \ddot{x}_{i}+\sum_{\forall j \in N_{i}} F_{i j}=F_{i}^{e}
$$

To obtain an exact solution of the differential equation (3), it has to be integrated in time. Various integration schemes have been tested [16] and Verlet integration emerged as the most suitable for application in MSS. Moreover, it is quite simple to implement. It discretizes time by replacing the derivatives by differences between sufficiently small steps dt.
The step dt is an additional parameter of the system which contributes heavily to its behaviour - a too small step will result in lengthier computations while too big steps will result in divergence of the integration scheme and therefore the system itself (i.e. it will not be able to achieve a stable position).

\section{EXPERIMENTS AND RESUTLS OF THE PHYSICS-BASED MODELING OF 3D VESSEL}

In order to testify our physical model of blood vessel and catheter, we designed a series of collision experiments between the virtual catheter and blood vessel model. As shown in Fig.6, we used the master side of novel robotic operating system to drive the virtual catheter to insert into the physics-based three dimensional model of blood vessel.

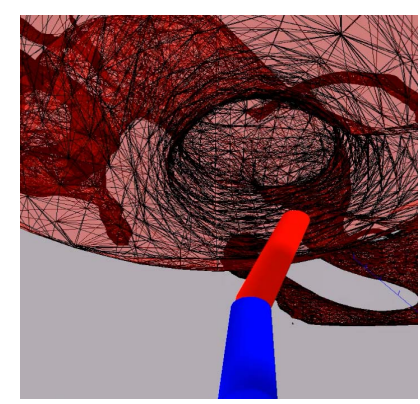

(a) $\mathrm{t}=0 \mathrm{~s}$

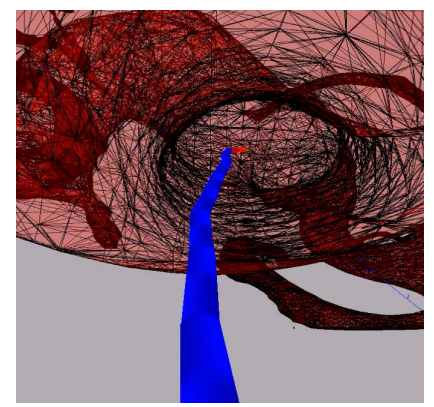

(c) $\mathrm{t}=0.4 \mathrm{~s}$

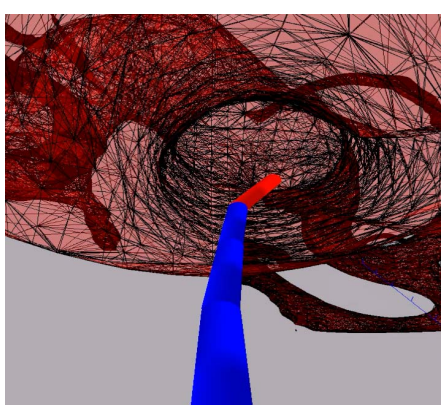

(b) $\mathrm{t}=0.2 \mathrm{~s}$

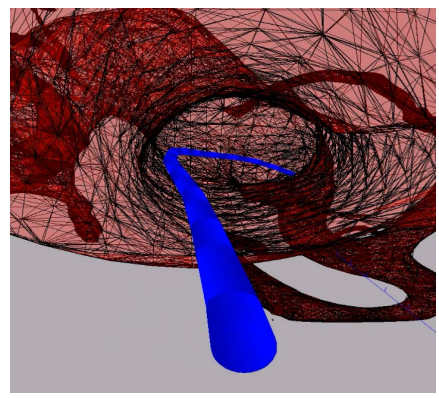

(d) $\mathrm{t}=0.6 \mathrm{~s}$
Fig. 7 The process of catheter inserting into the $3 \mathrm{D}$ Vessel

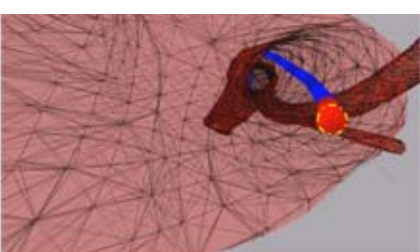

(1) $\mathrm{t}=0.8 \mathrm{~s}$

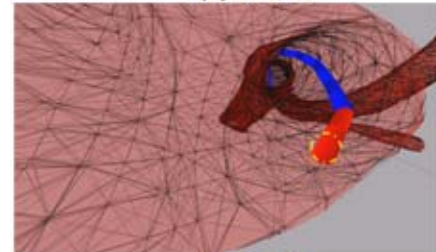

(3) $\mathrm{t}=1.2 \mathrm{~s}$

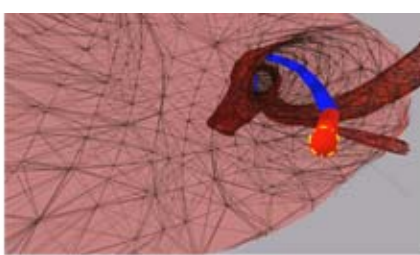

(2) $\mathrm{t}=1.0 \mathrm{~s}$

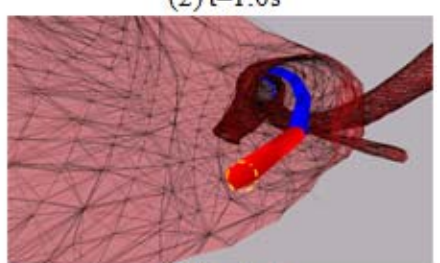

(4) $\mathrm{t}=1.4 \mathrm{~s}$
Fig. 8 The process of catheter rotating into the $3 \mathrm{D}$ Vessel 
The red part of the catheter is the tip top part which is used to collect the force information of the catheter when contact with the vessel. Fig.7 shows the process of rotating the catheter and move into the blood vessel model. We can detect the tip force in the VR system and sent it to the user from the master side.
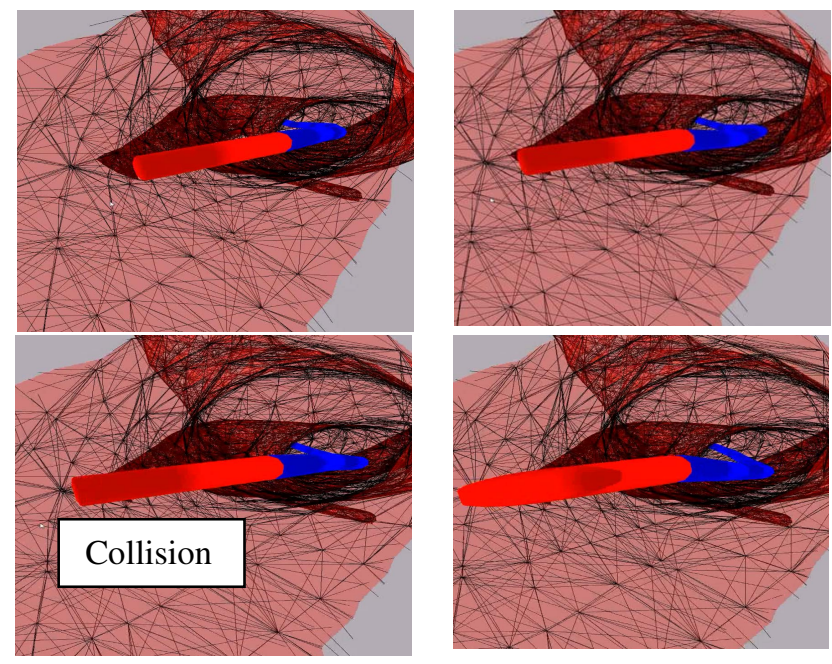

Fig.9 Deformation of the catheter and 3D blood vessel model when in the collision between each other

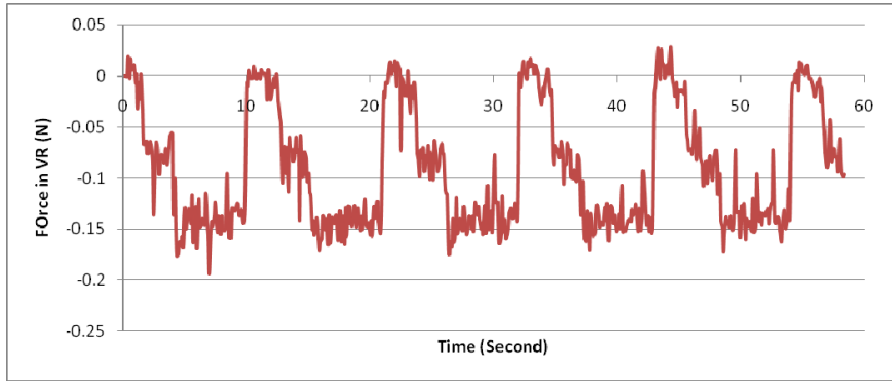

Fig.10 The calculated force in VR environment

Fig.9 show us the process of the collision of the catheter with the blood vessel model, and when collision happened, the deformation can be calculated in the VR system, and doctor can get the force information by the virtual feedback judge by the deformation or by the force sent by the master side.

In the VR system, we get the results which show that force trend line of the catheter-vessels interactions in virtual reality environment. There are many other complex interactions between the catheter and the vascular vessels and the process of deformation turns to be a little rigid, and it may generate calculation error of elastic force.

\section{CONCLUSION}

In this paper, we introduced a kind of virtual reality based training system which is used for the training of the interns to operate the Robotic Catheter System and improve the operation experience and auxiliary medical process. The novel robotic catheter operating system has good manoeuvrability, it can simulate surgeon's operating skill to insert and rotate catheter. The characteristic evaluations (rotating motion and inserting motion) have also been done to verify the validity of the system; the experimental results indicated that the stability and responsibility of system were good, the robotic catheter system was fitting to be used for training unskilled surgeons to do the operation of intravascular neurosurgery. The result of the work presented here is the virtual reality simulators allowing for the simulation of surgeon's operations for training in minimally invasive surgery based on our novel robotic catheter operating system. Based on the virtual model of catheter and blood vessels, we analyse and apply physical-based theory and implementation for these models. And the experimental results show that by defining the material properties of the catheter and the blood vessels, the behaviour of the catheter motion can be realistically simulated in a specific patient artery network, thereby allowing surgeons to train and rehearse new operative skills repeatedly.

In the future works, we will use the Virtual Reality System to help the unskilled surgeon improve their experience and make sure the safety of the operation by using the robotic catheter system.

\section{ACKNOWLEDGMENT}

This research is supported by Kagawa University Characteristic Prior Research fund 2012.

\section{REFERENCES}

[1] N. E. Seymour et al., "Virtual Reality Training Improves Operating Room Performance: Results of a Randomized, DoubleBlinded Study," Annals of Surgery, vol. 4, no. 236, 2002, pp. 458-464.

[2] A. G. Gallagher and C. U. Cates, "MD Approval of Virtual Reality Training for Carotid Stenting: What This Means for Procedural-Based Medicine," J. Am. Medical Assoc., vol. 292, no. 24, 2004, pp. $3024-3026$.

[3] J. Guo, N. Xiao, S. Guo, T. Tamiya: "Development of A Force Information Monitoring Method for A Novel Catheter Operating System" An International Interdisciplinary Journal of Information, Vol. 13, No.6, pp 1999- 2009, 2010.

[4] Filler, AG (2009): The history, development, and impact of computed imaging in neurological diagnosis and neurosurgery: CT, MRI, DTI: Nature Precedings DOI: 10.1038/npre.2009.3267.5

[5] F. Arai, M. Tanimoto, T. Fukuda, K. Shimojima, H. Matsuura, and M. Negoro. Distributed Virtual Environment for Intravascular Tele-Surgery Using Multimedia Telecommunication, Proceedings of VRAIS'96, pp. 79-85, 1996.

[6] M. Tanimoto, F. Arai, T. Fukuda, and M. Negoro. Augmentation of Safety in Teleoperation System for Intravascular Neurosurgery, Proceedings of the 1998 IEEE International Conference on Robotics \& Automation, pp.2890-2895, 1998. 
[7] C. Preusche, T. Ortmaier, G. Hirzinger, Teleoperation concepts in minimal invasive surgery. Control Engineering Practice, Vol.10, pp. 1245-1250, 2002.

[8] T. Fukuda, S. Guo, K. Kosuge, F. Arai, M. Negoro, and K. Nakabayash, Micro Active Catheter System with Multi Degree of Freedom, Proceedings of 1994 IEEE International Conference on Robotic and Automation, Vol.3, pp. 2290-2295, 1994.

[9] S. Guo, T. Nakamtra, T. Fukuda, K. Oguro, and M. Negoro, Micro Active Catheter Using ICPF Actuator Characteristic Evaluation, Proceedings of IEEE the 22nd Annual International Conf. on Industrial Electronics, Control, and Instrumentation, pp.1312-1317, 1996.

[10] F. Arai, R. Fujimura, T. Fukuda, and M. Negoro, New Catheter Driving Method Using Linear Stepping Mechanism for Intravascular Neurosurgery. Proceedings of the 2002 IEEE International Conference on Robotics \& Automation, pp. 29442949, 2002.

[11] W. Feng, S. Guo, C. Chi, H. Wang, K. Wang and X. Ye, Realization of a Catheter Driving Mechanism with Micro tactile sensor for Intravascular Neurosurgery. Proceedings of the 2006 IEEE International Conference on Robotics and Biomimetics, pp1628-1633, 2006.

[12] W. Feng, C. Chi, H. Wang, K. Wang, X. Ye and S. Guo. Highly Precise Catheter Driving Mechanism for Intravascular Neurosurgery. Proceedings of 2006 IEEE International Conference on Mechatronics and Automation, pp 990-995, 2006.

[13] S. Guo, H. Kondo, J. Wang, J. Guo, T. Tamiya. A New Catheter Operationg System for Medical Applications. Proceedings of the 2007 ICME International Conference on Complex Medical Engineering, pp. 82-87, 2007.

[14] S. Ikeda, F. Arai, T. Fukuda, M. Negoro, K.Irie, and I. Takahashi, et. al., In Vitro Patient-Tailored Anatomaical Model of Cerebral Artery for Evaluating Medical Robots and Systems for Intravascular Neurosurgery, proceedings of 2005 IEEE/RSJ International Conference on Intelligent Robots and Systems, pp. 1558-1563, 2005.

[15] J. Peirs, J. Clijnen, D. Reynaerts, H. V. Brussel, P. Herijgers, B. Corteville, et. al., A micro optical force sensor for force feedback during minimally invasive robotic surgery, Sensors and Actuators, Vol.115, No.2-3, pp.447-455, 2004.

[16] R. Sedaghati, J. Dargahi, H. Singh. Design and modeling of an endoscopic piezoelectric tactile sensor, International Journal of Solids and Structures, Vol.42, No.21-22, pp.5872-5886, 2005.

[17] Y. Thakui, J. S. Bax, D. W. Holdsworth and M. Drangova, Design and Performance Evaluation of a Remote Catheter Navigation System, IEEE Transactions on biomedical engineering, vol.56, No. 7, pp. 1901-1908, 2009.

[18] Y. Fu, A. Gao, H. Liu, and S. Guo, The master-slave catheterisation system for positioning the steerable catheter, Int. J. Mechatronics and Automation, Vol. 1, Nos. 3/4, pp. 143-152, 2011.

[19] J. Wang and S. Guo, H. Kondo, J. Guo and T. tamiya, A Novel Catheter Operating System with Force Feedback for Medical Applications, International Journal of Information Acquisition, (2008)5: pp.83-91,2008.

[20] Y. C. Wu, J. S. Chen, Toward the identification of EMG-signal and its bio-feedback application, International Journal of Mechatronics and Automation, vol. 1, No.2, pp.112-120, 2011.

[21] Y. Fu, H. Liu, S. Wang, et al, Skeleton based active catheter navigation, The International Journal of Medical Robotics and Computer Assisted Surgery, vol. 5, No. 2, pp. 125-135, 2009.

[22] Nan Xiao, Jian Guo, Shuxiang Guo, and Takashi Tamiya, A Robotic Catheter System with Real-time Force Feedback and
Monitor, Journal of Australasian Physical and Engineering Sciences in Medicine, Vol. 35, No. 3, pp. 283-289, 2012.

[23] Jian Guo, Shuxiang Guo, Nan Xiao, Xu Ma, Shunichi Yoshida, Takashi Tamiya and Masahiko Kawanishi, "A Novel Robotic Catheter System with Force and Visual Feedback for Vascular Interventional Surgery", International Journal of Mechatronics and Automation, Vol.2, No.PP., 2012.

[24] Jin Guo, Shuxiang Guo, Nan Xiao, and Baofeng Gao, Virtual Reality Simulators based on a Novel Robotic Catheter Operating system for Training in Minimally Invasive Surgery, Journal of Robotics and Mechatronics, Vol. 24, No. 4, pp. 649-655, 2012.

[25] Xu Ma, Shuxiang Guo, Nan Xiao, Jian Guo, Shunichi Yoshida, Takashi Tamiya, and Masahiko Kawanishi, Development of a Novel Robotic Catheter Manipulating Systemwith Fuzzy PID Control, International Journal of Intelligent Mechatronics and Robotics (IJIMR), Vol. 2, No. 2, pp. 58-77, 2012.

[26] Yili Fu, Anzhu Gao, Hao Liu, and Shuxiang Guo, The masterslave catheterisation system for positioning the steerable catheter, Int. J. Mechatronics and Automation, Vol. 1, Nos. 3/4, pp. 143$152,2011$. 